\title{
Response to: "Letter to the Editor: Is Low-Volume Disease in the Sentinel Node After Neoadjuvant Chemotherapy an Indication for Axillary Dissection? Miscalculation of Sensitivity and False Negative Rate"
}

\author{
Tracy-Ann Moo, MD, and Monica Morrow, MD \\ Breast Service, Department of Surgery, Memorial Sloan Kettering Cancer Center, New York, NY
}

We appreciate the time and effort Drs. Smiley and Castaldi have put into analysis of the results reported in our article entitled Is Low-Volume Disease in the Sentinel Node After Neoadjuvant Chemotherapy an Indication for Axillary Dissection? Upon review with our statistician, we agree there was an error in the calculation of the falsenegative rate and sensitivity for the intraoperative frozen section procedure. As pointed out, the correct false-negative rate for frozen section is $15.4 \%$ and the correct sensitivity is $84.6 \%$, instead of the reported $6.2 \%$ and $93.8 \%$, respectively. We have submitted a correction to Annals of Surgical Oncology for publication.

With respect to the relationship between disease volume in the sentinel node and the likelihood of having additional non-sentinel node metastases at axillary dissection, the false-negative rate and sensitivity of the frozen-section procedure are not relevant and were not used for these calculations. We found that regardless of the method of detection (frozen section vs. permanent section), among patients with a positive sentinel node, those with micrometastases were as likely as those with macrometastases to have additional positive non-sentinel nodes at axillary dissection, i.e., $64 \%$ vs. $62 \%$. Therefore, axillary dissection is indicated for both sentinel lymph node micrometastases and macrometastases after neoadjuvant chemotherapy.

(C) Society of Surgical Oncology 2020

First Received: 5 February 2020;

Published Online: 27 February 2020

T.-A. Moo, MD

e-mail: moot@mskcc.org
Drs. Smiley and Castaldi suggest calculation of the sensitivity and false-negative rate for frozen section based on sentinel node metastasis size (in Table 2 of their Letter to the Editor). We agree that their calculation demonstrates that the false-negative rate of frozen section decreases with increasing sentinel node metastasis size, a result which is consistent with prior studies, ${ }^{1,2}$ and addressed in the Introduction and Discussion sections of our article. There is a slight error in their calculation of the false-negative rate for isolated tumor cells, which should be $77 \%$ rather than $87 \%$. The overall number of isolated tumor cells in our study was too small to draw conclusions regarding the false-negative rate. In addition, immunohistochemistry is not routinely performed at our institution, and so the number of isolated tumor cells reported in our article does not reflect the true incidence.

Sincerely,

Tracy-Ann Moo, MD

DISCLOSURES Dr. Monica Morrow has received speaking honoraria from Genomic Health. Dr. Tracy-Ann Moo has no conflict of interest disclosures to report.

\section{REFERENCES}

1. Rubio IT, Aznar F, Lirola J, Peg V, Xercavins J. Intraoperative assessment of sentinel lymph nodes after neoadjuvant chemotherapy in patients with breast cancer. Ann Surg Oncol. 2010;17(1):235-9.

2. Komenaka IK, Torabi R, Nair G, et al. Intraoperative touch imprint and frozen section analysis of sentinel lymph nodes after neoadjuvant chemotherapy for breast cancer. Ann Surg. 2010;251(2):319-22.

Publisher's Note Springer Nature remains neutral with regard to jurisdictional claims in published maps and institutional affiliations. 